\title{
Processo de Gestão de Processos: uma implementação da gerência de processos organizacionais do CMMI-SW Nível 3
}

\author{
Tatiana Monteiro, Fabiana Marinho, Márcia Sampaio, \\ Solange Araújo, Carlo Giovano S. Pires
}

Instituto Atlântico, Rua Chico Lemos, 946, 60822-780, Fortaleza - Ceará

\{tatiana, fabiana, marcia, solange, cgiovano\}@atlantico.com.br

\begin{abstract}
In the CMMI-SW level 3, the process concept is largely introduced in the organization. However, the most of the organizations uses a proprietary informal description in the definition of its processes, making it difficult the agreement and implementation of them. The definition of architecture and a process that leads this structure is critical for the organization of the processes and the success of the organizational improvement program. In this context, this work shows the experience of the Instituto Atlantico in the establishment of the Process of Management of Processes.
\end{abstract}

Resumo: No CMMI-SW nível 3 o conceito de processo é fortemente introduzido na organização. No entanto, a maioria das organizações utiliza uma descrição proprietária informal na definição de seus processos, dificultando o entendimento e implementação dos mesmos. A definição de uma arquitetura e de um processo que conduza esta estruturação é fundamental para a organização dos processos e para o sucesso do programa de melhoria organizacional. Neste contexto, este trabalho mostra a experiência do Instituto Atlântico no estabelecimento do Processo de Gestão de Processos.

\section{Introdução}

A preocupação com a qualidade do processo de software está se tornando tão essencial para as organizações quanto a qualidade dos produtos entregues aos clientes. Como a qualidade dos produtos de software está diretamente relacionada com a qualidade do seu processo de desenvolvimento, muitas empresas passaram a investir na melhoria de seus processos. O CMMI (Capability Maturity Model Integration) é um modelo desenvolvido pelo Software Engineering Institute (SEI) que busca orientar as organizações de software na implementação de melhorias contínuas em seu processo de desenvolvimento.

Este artigo apresenta a experiência do Instituto Atlântico, uma organização avaliada em fevereiro de 2006 como nível 3 do CMMI-SW, no estabelecimento do processo de gestão de processos que implementa as áreas de processo de Organizational Process Focus (OPF) e Organizational Process Definition (OPD) do modelo CMMISW.

Este trabalho está organizado em sete seções. Na seção 2, apresentamos os conceitos das áreas de processo do CMMI relacionadas à definição e manutenção dos processos. Descrevemos a estrutura da arquitetura de processos do Instituto Atlântico na seção 3. Na seção 4, descrevemos o processo de criação do Engineering Process Group (EPG) do Atlântico. Na seção 5, apresentamos o mapeamento entre o processo de gestão de processos e as práticas específicas e genéricas de OPD e OPF. A Base de 
Dados Organizacional é descrita na seção 6 e na seção 7, apresentamos as conclusões do nosso trabalho.

\section{A gestão de processos no CMMI-SW}

No CMMI-SW nível 3, os processos são documentados, padronizados e integrados em um processo padrão da organização, que pode ser adaptado de acordo com as necessidades de cada projeto [SEI, 2002] e [Kulpa, 2003]. Pertencem a este nível de maturidade as áreas de processo (Process Areas - PA's) de Organizational Process Definition (OPD) e Organizational Process Focus (OPF) que abordam as questões de definição e manutenção dos processos.

O objetivo da PA de OPF é planejar e implementar as melhorias de processos no nível organizacional baseada no entendimento dos pontos fortes e fracos dos processos organizacionais e seus ativos. Também envolve criar e manter um entendimento dos processos dos projetos, bem como coordenar as atividades para avaliar, desenvolver, manter e melhorar esses processos. As metas definidas para esta PA [Sommerville, 2001] estão descritas a seguir: (i) alinhar os objetivos e necessidades dos processos com as metas e objetivos organizacionais, (ii) coordenar as atividades de desenvolvimento e melhoria de processo em toda a organização, (iii) identificar os pontos fortes e as oportunidades de melhoria dos processos identificados e $(i v)$ planejar as atividades de desenvolvimento e melhoria de processo no nível organizacional.

Esta área de processo exige a criação do EPG (Engineering Process Group) que é o grupo responsável por estudar, implementar e manter os processos da organização.

O objetivo da PA de OPD é desenvolver e manter um conjunto de ativos de processo que sejam úteis e melhorem o desempenho dos processos utilizados nos diversos projetos. Visa também, estabelecer uma base sólida capaz de gerar benefícios acumulativos e de longo prazo para toda a organização. As metas definidas para esta PA [Sommerville, 2001] estão descritas a seguir: $(i)$ estabelecer e manter um processo de software padrão para a organização, (ii) estabelecer e manter critérios de adaptação para os processos utilizados pelos projetos, (iii) estabelecer e manter ciclos de vida padrão da organização, (iv) coletar, revisar e disponibilizar em um repositório organizacional as informações relativas ao uso do processo padrão da organização e $(v)$ estabelecer e manter um repositório contendo as medições da organização.

\section{Arquitetura de processos do Instituto Atlântico}

A arquitetura de processos do Atlântico foi estruturada de maneira a refletir diretamente as práticas dos níveis 2 e 3 do CMMI-SW e da norma ISO 9001:2000, sendo constituída das áreas organizacional (3 processos), gestão de projetos (5 processos), suporte (5 processos), engenharia (10 processos) e administrativo-financeira (7 processos), conforme mostrado na Figura 1. A área organizacional agrupou os processos relacionados à definição e manutenção dos processos organizacionais e a gestão do programa de treinamento. A área de gestão de projetos foi composta pelos processos necessários para a gerência e controle dos projetos. A área de engenharia agrupou os processos utilizados no desenvolvimento dos produtos de software e hardware. A área administrativo-financeira agrupou os processos necessários para as atividades da gerência administrativo-financeira da organização. A área de suporte foi composta pelos processos responsáveis por dar suporte às atividades executadas nos demais processos. 


\section{Sistema de Gestão da Qualidade}

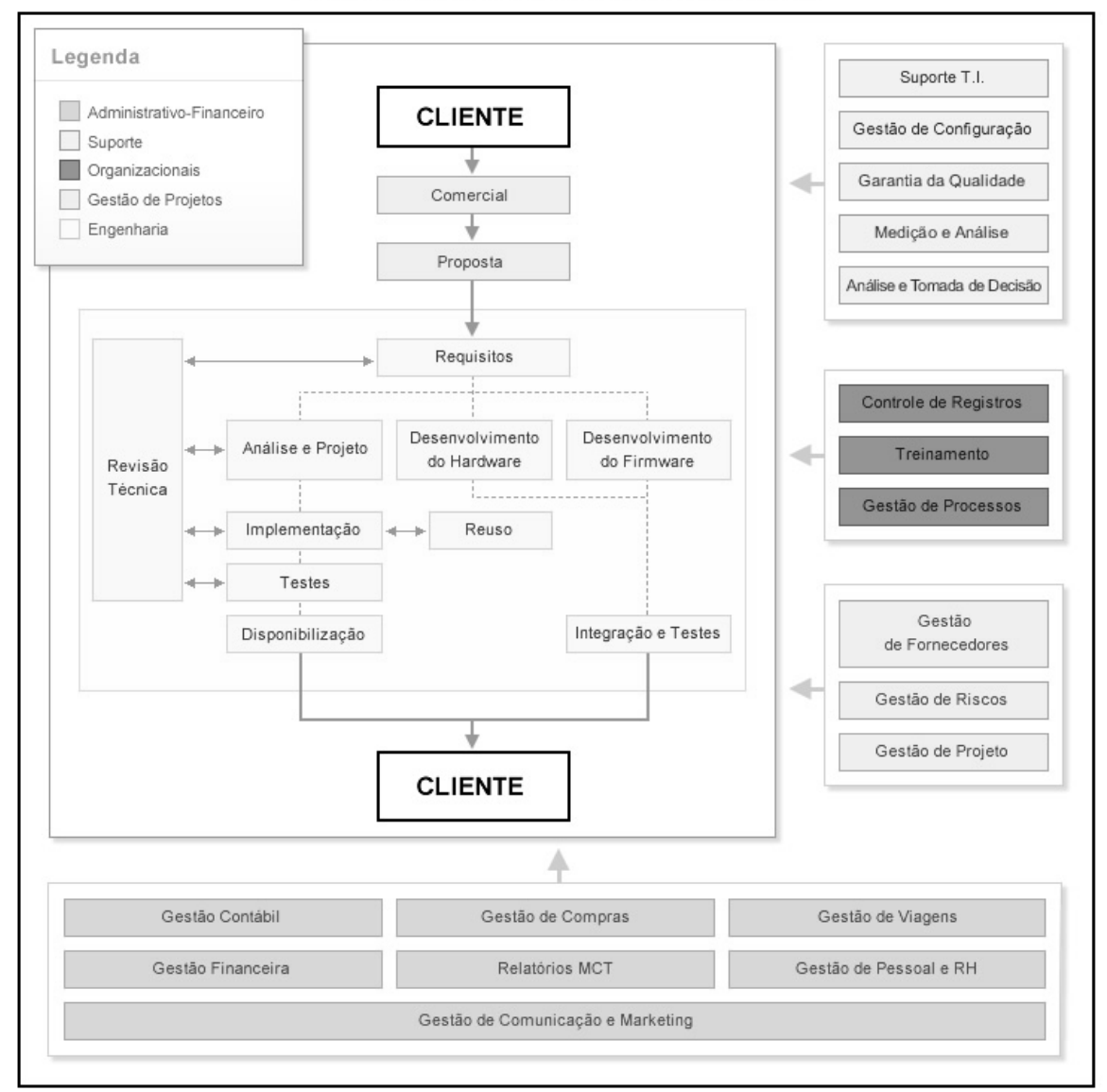

Figura 1 - Arquitetura de processos do Instituto Atlântico

Os processos foram definidos com base na arquitetura de processos disponibilizada pelo RUP [Kruchten, 2003]. O RUP foi escolhido por fornecer uma notação formal para a definição de processos, uma abordagem disciplinada para atribuir tarefas e responsabilidades e utilizar a notação da UML [Rumbaugh, Booch, Jacobson, 2000]. Além disso, é amplamente utilizado e difundido, facilitando o entendimento da estrutura e notações do processo. Nesse contexto, os processos do Instituto Atlântico são definidos através de um modelo orientado a objetos e descritos com o uso de UML, de uma forma similar à modelagem de software. Cada processo é descrito por meio de fluxos de trabalho e outros elementos. Os fluxos de trabalho correspondem a um diagrama de atividades da UML e mostram uma seqüência significativa de grupos de atividades que produz um resultado determinado. Cada grupo de atividades é descrito por um diagrama de detalhamento de fluxo de trabalho. Esses diagramas mostram todas as atividades do grupo, os papéis envolvidos e os artefatos de entrada e saída. Os outros elementos do processo são descritos nos formatos de templates, relatórios, orientações de trabalho, pontos de verificação, mentor de ferramentas e conceitos.

A seguir, relatamos a experiência de criação do Engineering Process Group (EPG) do Instituto Atlântico responsável pela definição, documentação e manutenção da arquitetura de processos organizacionais. 


\section{Engineering Process Group - EPG}

O Engineering Process Group (EPG) do Instituto Atlântico foi constituído inicialmente por integrantes alocados parcialmente e agrupados em Process Area Teams (PAT's). O objetivo destes PAT's era definir os processos a partir de contribuições e visões diferenciadas das diversas áreas da instituição.

Os PATs foram classificados em categorias de acordo com as áreas de processo definidas na arquitetura de processos da organização e eram estruturados nos papéis de líder, membro permanente, consultor, membro $A d$ Hoc e redator. Cada PAT possuía um cronograma detalhado das suas atividades que era acompanhado pelo líder.Todos os processos elaborados pelos PAT's foram validados com os responsáveis pela execução dos mesmos e as sugestões de melhoria foram incorporadas. Neste momento, o número de integrantes do EPG foi reduzido. Para cada processo foi alocado um responsável e as atividades do grupo passaram a serem acompanhadas por um único coordenador.

O acompanhamento das atividades do EPG é realizado através de reuniões mensais, nas quais são tratadas as questões de acompanhamento do programa de melhoria, avaliação de sugestões de melhoria nos processos, acompanhamento dos riscos relacionados ao programa de melhoria, alocação de membros do EPG, dentre outras questões. Também foram institucionalizados dois níveis de reunião de acompanhamento com a alta direção. No primeiro nível participam o gerente da área de Suporte ao Processo de Desenvolvimento (SPD) e o coordenador do EPG. Esta reunião tem como objetivo o relato e o acompanhamento do progresso das atividades do processo de gestão de processos. Uma segunda reunião conta com a participação do Gerente do SPD e do Superintendente e tem como objetivo o relato das atividades do processo de gestão de processos para o nível executivo.

As atividades do EPG são verificadas através das avaliações de qualidade organizacionais. Estas avaliações são realizadas pelo grupo de garantia da qualidade. $\mathrm{O}$ objetivo destas avaliações é garantir que o processo de gestão de processos seja executado em conformidade com as PA's de OPD e OPF do modelo CMMI-SW.

A seguir, apresentamos o processo de gestão de processos do Atlântico e mapeamos o processo criado para as áreas de processo de OPD e OPF do modelo CMMI-SW.

\section{O processo de Gestão de Processos}

$\mathrm{O}$ processo de gestão de processos do Instituto Atlântico é apresentado na Figura 2. O fluxo das atividades descreve desde o alinhamento dos objetivos e necessidades organizacionais até a manutenção e estabelecimento dos repositórios (Base de Dados Organizacional e Repositório Organizacional de Medições).

$\mathrm{Na}$ Tabela 1 é apresentado o mapeamento entre as macroatividades do processo de gestão de processos e as práticas requeridas pelas PA's de OPD e OPF do modelo CMMI-SW. Conforme podemos verificar, quase todas as práticas de OPD e OPF do CMMI-SW foram atendidas pelo processo de gestão de processos apresentado. As demais práticas são atendidas por outros processos e pela política, de acordo com a Tabela 2. 


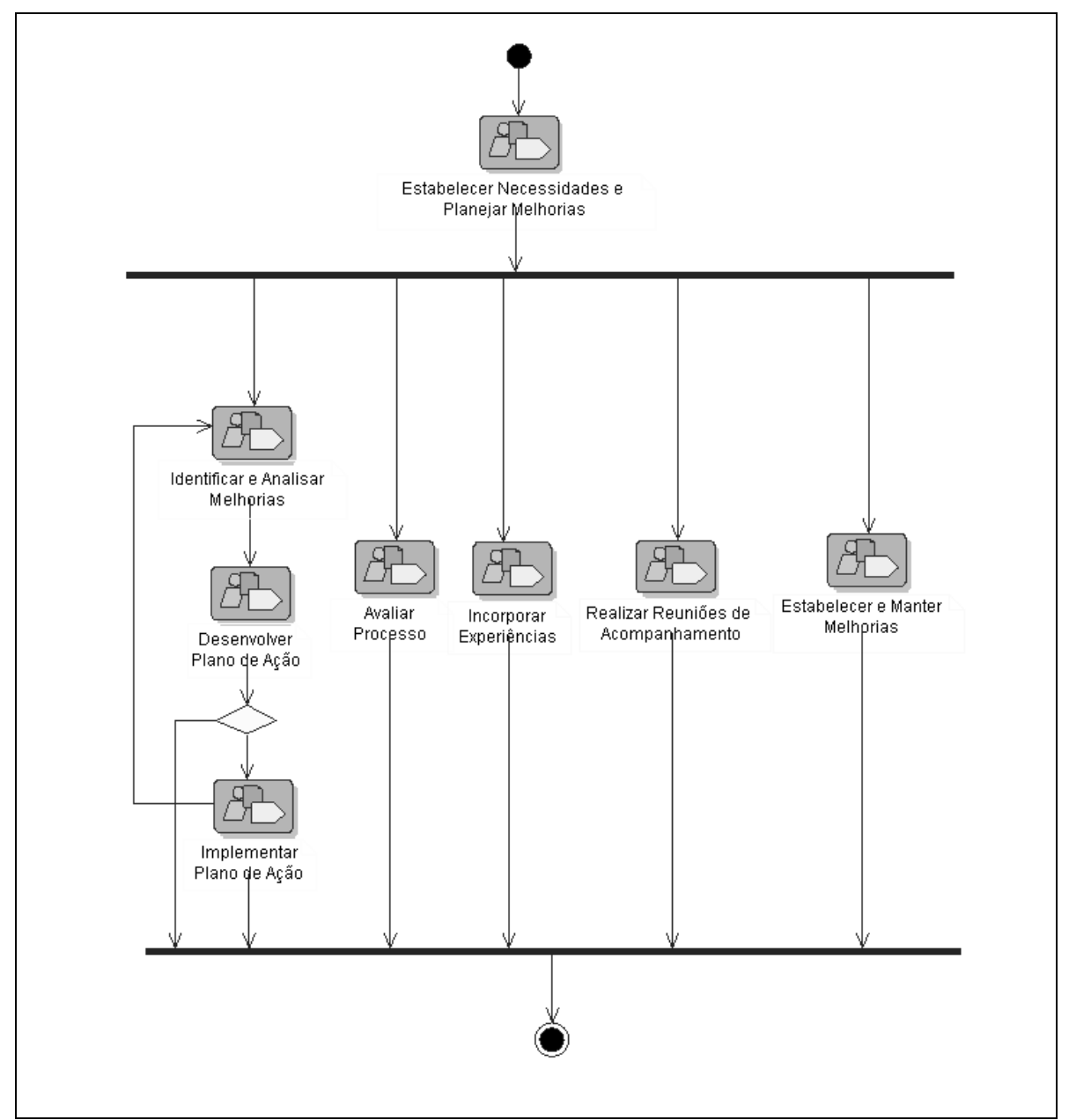

Figura 2-Fluxo de trabalho do processo de gestão de processo

Tabela 1 - Mapeamento entre as macroatividades do processo e as práticas do CMMI-SW

\begin{tabular}{|c|c|c|}
\hline Macroatividade & Finalidade & Prática CMMI-SW \\
\hline $\begin{array}{l}\text { Estabelecer } \\
\text { Necessidades e } \\
\text { Planejar melhoria }\end{array}$ & $\begin{array}{l}\text { Estabelecer e manter as necessidades } \\
\text { dos processos e planejar o programa de } \\
\text { melhoria. As necessidades dos } \\
\text { processos devem estar alinhas as metas } \\
\text { e objetivos organizacionais. }\end{array}$ & $\begin{array}{l}\text { (OPF) SP } 1.1 \text { - Estabelecer as } \\
\text { necessidades do processo } \\
\text { organizacional } \\
\text { (OPF e OPD) GP } 2.2 \text { - Planejar o } \\
\text { processo } \\
\text { (OPF e OPD) GP } 2.3 \text { - Prover } \\
\text { recursos } \\
\text { (OPF e OPD) GP } 2.4 \text { - Atribuir } \\
\text { responsabilidades } \\
\text { (OPD e OPF) GP } 2.7 \text { - Identificar e } \\
\text { envolver stakeholders relevantes }\end{array}$ \\
\hline
\end{tabular}




\begin{tabular}{|c|c|c|}
\hline $\begin{array}{l}\text { Identificar e } \\
\text { Analisar melhorias }\end{array}$ & $\begin{array}{l}\text { Identificar as melhorias nos processos } \\
\text { através de: solicitações dos } \\
\text { colaboradores, pontos identificados em } \\
\text { avaliações CMMI (classe A, B, C e } \\
\text { interna), auditorias ISO (oficial e } \\
\text { manutenção), } \\
\text { organizacionais. O EPG é responsável } \\
\text { por viabilizar as incorporações das } \\
\text { sugestões de melhorias que forem } \\
\text { pertinentes. }\end{array}$ & $\begin{array}{l}\text { (OPF) SP } 1.3 \text { - Identificar melhorias } \\
\text { no processo organizacional } \\
\text { (OPD e OPF) GP } 3.2 \text { - Coletar } \\
\text { informações de melhorias }\end{array}$ \\
\hline $\begin{array}{l}\text { Desenvolver plano } \\
\text { de ação }\end{array}$ & $\begin{array}{l}\text { Elaborar planos de ação para alinhar os } \\
\text { processos aos modelos adotados e aos } \\
\text { objetivos de negócio da organização. }\end{array}$ & $\begin{array}{l}(\mathrm{OPF}) \mathrm{SP} 2.1 \text { - Estabelecer planos de } \\
\text { ação para o processo }\end{array}$ \\
\hline $\begin{array}{l}\text { Implementar Plano } \\
\text { de Ação }\end{array}$ & $\begin{array}{l}\text { Implementar os planos de ação, } \\
\text { executar os projetos pilotos e } \\
\text { disponibilizar os ativos organizacionais. }\end{array}$ & $\begin{array}{l}\text { (OPF) SP } 2.2 \text { - Implementar plano de } \\
\text { ação para o processo } \\
\text { (OPF) SP } 2.3 \text { - Disponibilizar os } \\
\text { ativos do processo organizacional }\end{array}$ \\
\hline Avaliar Processo & $\begin{array}{l}\text { Planejar e realizar avaliações CMMI } \\
\text { (classe A, B, C e interna), auditorias } \\
\text { ISO (oficial e manutenção) e avaliações } \\
\text { relacionadas aos } \\
\text { organizacionais (por } r \text { exemplo, } \\
\text { treinamento, gestão de processos e } \\
\text { medição e análise). }\end{array}$ & $\begin{array}{l}\text { (OPF) SP } 1.2-\text { Avaliar os processos } \\
\text { organizacionais } \\
\text { (OPD e OPF) GP } 2.9-\text { Avaliar a } \\
\text { aderência de forma objetiva }\end{array}$ \\
\hline $\begin{array}{l}\text { Incorporar } \\
\text { Experiência }\end{array}$ & $\begin{array}{l}\text { Incorporar no processo organizacional } \\
\text { as experiências obtidas nos projetos } \\
\text { passados, sugestões de melhorias, } \\
\text { medições relevantes aos processos. }\end{array}$ & $\begin{array}{l}\text { (OPF) SP } 2.4 \text { - Incorporar } \\
\text { experiências relatadas do processo } \\
\text { nos ativos de processo organizacional } \\
\text { (OPD e OPF) GP } 3.2 \text { - Coletar } \\
\text { informações de melhorias }\end{array}$ \\
\hline $\begin{array}{l}\text { Realizar Reuniões } \\
\text { de } \\
\text { Acompanhamento }\end{array}$ & $\begin{array}{l}\text { Realizar reuniões de acompanhamento } \\
\text { do programa de melhoria. }\end{array}$ & $\begin{array}{l}\text { (OPD e OPF) GP } 2.8 \text { - Monitorar e } \\
\text { controlar o processo. } \\
\text { (OPD e OPF) GP } 2.10 \text { - Revisar o } \\
\text { status com a gerência de alto nível }\end{array}$ \\
\hline $\begin{array}{l}\text { Estabelecer e } \\
\text { Manter Melhorias }\end{array}$ & $\begin{array}{l}\text { Estabelecer e manter: os modelos de } \\
\text { ciclos de vida organizacionais, a } \\
\text { arquitetura de processos, os ativos } \\
\text { organizacionais, os critérios de } \\
\text { adaptação, a Base de Dados } \\
\text { Organizacional e o Repositório } \\
\text { Organizacional de Medições. }\end{array}$ & $\begin{array}{l}\text { (OPD) SP } 1.1 \text { - Estabelecer os } \\
\text { processos padrão } \\
\text { (OPD) SP } 1.2 \text { - Estabelecer } \\
\text { descrições dos modelos de ciclo de } \\
\text { vida } \\
\text { (OPD) SP } 1.3 \text { - Estabelecer critérios } \\
\text { de adaptação e guias } \\
\text { (OPD) SP } 1.4 \text { - Estabelecer o } \\
\text { repositório organizacional de } \\
\text { medição } \\
\text { (OPD) SP } 1.5 \text { - Estabelecer a base de } \\
\text { dados de ativos do processo } \\
\text { organizacional }\end{array}$ \\
\hline
\end{tabular}

Tabela 2. Mapeamento entre as práticas do CMMI-SW e as políticas e processos

\begin{tabular}{|l|l|}
\hline \multicolumn{1}{|c|}{ Prática CMMI } & \multicolumn{1}{c|}{ Política/Processo } \\
\hline $\begin{array}{l}\text { (OPD e OPF) GP 2.1 - Estabelecer uma política } \\
\text { organizacional }\end{array}$ & Políticas Institucionais da Qualidade \\
\hline
\end{tabular}




\begin{tabular}{|l|l|}
\hline (OPD e OPF) GP 2.5 - Treinar as pessoas & Processo de Treinamento \\
\hline (OPD e OPF) GP 2.6 - Gerenciar as configurações & $\begin{array}{l}\text { Processo de Gestão de Configuração e } \\
\text { Mudança }\end{array}$ \\
\hline $\begin{array}{l}\text { (OPD e OPF) GP } 2.9 \text { - Avaliar a aderência de forma } \\
\text { objetiva }\end{array}$ & Processo de Garantia da Qualidade \\
\hline $\begin{array}{l}\text { (OPD e OPF) GP } 3.1 \text { - Estabelecer um processo } \\
\text { definido }\end{array}$ & Processos e Processo de Gestão de Projetos \\
\hline
\end{tabular}

Uma Base de Dados Organizacional foi estabelecida na intranet do Instituto Atlântico para dar suporte à execução do processo de gestão de processos. Esta base de dados é descrita a seguir.

\section{Base de Dados Organizacional do Instituto Atlântico}

A Base de Dados Organizacional do Instituto Atlântico disponibiliza para todos os colaboradores da instituição informações e ferramentas relevantes para o sistema de gestão da qualidade, tais como, políticas institucionais, repositório de processos, repositório de medições, ferramenta de sugestão de melhorias, o programa de treinamentos, catálogo de reuso, melhores documentos e lições aprendidas. A seguir estão descritas as principais ferramentas que compõem a base de dados.

- Repositório Organizacional de Gestão do Conhecimento: ferramenta (MC2 [Secrel, 2006]) onde estão armazenados os indicadores que formam a base histórica, os indicadores organizacionais e os indicadores estratégicos; Armazena também os melhores documentos, artigos, dados e lições aprendidas de projetos, fornecendo um mecanismo de busca por palavras-chave.

- Repositório de Processos: site navegável gerado a partir do Modelo Rational Rose/RPW [IBM Corporation, 2004] do processo contendo toda a arquitetura de processos, diagramas, procedimentos, templates e orientações.

- Site do Sistema de Gestão da Qualidade: (SharePoint [Microsoft Corporation, 2006]) repositório que armazena os artefatos dos processos organizacionais, como, plano de melhoria, plano de treinamento, plano de medição e análise, especificações das medições, planejamento e resultados das avaliações CMMI, plano de gestão de configuração e mudança organizacional.

- Ferramenta de Sugestão de Melhorias: ferramenta (JIRA [Atlassian, 2005]) onde são registradas as sugestão de melhorias e problemas encontrados nos processos. Além disso, é utilizada para a submissão de lições aprendidas e melhores documentos dos projetos (best documents).

- Ferramenta de Gestão de Treinamento: ferramenta (MC2 [Secrel, 2006]) que gerencia as competências organizacionais, catálogo de treinamentos, agenda de treinamentos, avaliação da execução do treinamento, listas de participação, provas eletrônicas e material utilizado nos treinamentos.

Além das ferramentas acima, a Base de Dados Organizacional disponibiliza um projeto conceito denominado Sócrates. Este projeto documenta indicadores, que representam as médias organizacionais, e lições aprendidas da organização. Além o disso, o projeto Sócrates descreve pontos comuns de melhoria levantados em reuniões de final de fase nos projetos, ou seja, apresenta um consolidado de melhores práticas da instituição e pontos fracos identificados. 
As informações armazenadas no projeto Sócrates são utilizadas pelos demais projetos do Atlântico como referencial para a realização das atividades de planejamento.

A Base de Dados Organizacional também possibilita a consulta aos dados de outros projetos já concluídos no Instituto Atlântico que servem como base para a realização de novos projetos. A busca destes dados é parametrizada conforme a característica do projeto. A consulta utilizando esta parametrização pode resultar tanto dados de projetos (lições aprendidas e indicadores) como em melhores documentos de projetos que possuam as mesmas características.

\section{Conclusão}

Esse artigo apresentou a experiência do Instituto Atlântico na definição e institucionalização do Processo de Gestão de Processos alinhado as áreas de processos organizacionais do CMMI-SW Nível 3: OPD e OPF. O estabelecimento deste processo apresentou os seguintes benefícios:

- definição de um arquitetura modularizada por áreas de processo;

- representação formal de todos os processos com base no RUP e na UML;

- uniformidade na estrutura e representação dos processos CMMI-SW nível 3;

- base de dados organizacional através de ambiente Web de acesso facilitado e integrado com as demais ferramentas;

- Navegabilidade amigável nos processos organizacionais;

- Armazenamento e procura de melhores documentos, boas práticas, medições com base em características de projetos e outras palavras-chave;

O estabelecimento de ferramentas para dar suporte ao processo de gestão de processo foi fundamental para a rápida implantação e disponibilização dos ativos organizacionais.

\section{Referências Bibliográficas}

Sommerville, Ian. Software Engineering, 6th Edition, Addison-Wesley Publishers Ltd., 2001.

SEI - Software Engineering Institute. Capability Maturity Model Integration, version 1.1. CMMI for Software Engineering (CMMI-SW/IPPD, v1.1) Staged Representation. - Software Engineering Institute, 2002.

Kulpa, Margaret K.; JOHNSON, Kent A., Interpreting the CMMI: a process improvement approach. - Florida, 2003.

Atlassian Software Systems Pty Ltd. All rights reserved. Copyright (C) 2005. http://www.atlassian.com/software/jira/. Acessado em 23/02/06.

Secrel consultoria e sistemas Ltd. All rights reserved. http://www.mc2.com.br . Acessado em 23/02/06.

Kruchten, Philippe. Rational Unified Process made easy: A practioner's guide to the RUP, Addison-Wesley Publishers Ltd. 2003.

Rumbaugh, J., Booch, G., Jacobson, I. UML Guia do Usuário. Editora Campus. 2000.

Microsoft Corporation. All rights reserved. Copyright (C) 2006. http://www.microsoft.com/brasil/office/sharepoint/default.asp. Acessado em 23/02/06.

IBM Corporation. All Rights Reserved. Copyright (C) 2004 http://www128.ibm.com/developerworks/rational/library/6001.html. Acessado em 23/02/06. 